\title{
Computação e Sociedade: Uma Proposta de Educação em Computação para o Oitavo Ano do Ensino Fundamental II
}

\author{
Bianca L. Santana ${ }^{1}$, Luis Gustavo J. Araujo ${ }^{1}$, Roberto A. Bittencourt ${ }^{1}$ \\ ${ }^{1}$ UEFS - Universidade Estadual de Feira de Santana \\ Av. Transnordestina, s/n, Novo Horizonte \\ Feira de Santana - BA, Brasil - 44036-900 \\ \{biancasantana.ls, luisaraujo.ifba\}@gmail.com, roberto@uefs.br
}

\begin{abstract}
This work presents a proposal of curriculum and a textbook for the eighth grade of middle school, based on guidelines and reference curricula from the field of computing education. These materials aim to contribute to popularize computing in basic education. The book combines the use of collaborative systems, reflection on online social networks, educational robotics, block-based programming, and an introduction to a text-based programming language, using contexts that provide immediate feedback and a spiral learning approach.
\end{abstract}

Resumo. Este trabalho apresenta uma proposta curricular e um livro didático de computação para o oitavo ano do ensino fundamental II brasileiro, baseados em diretrizes e currículos de referência da área. Estes materiais objetivam contribuir para a popularização da computação na educação básica. O livro combina o uso de sistemas colaborativos, uma reflexão sobre redes sociais online, robótica educacional, programação através de linguagens de blocos e uma introdução a uma linguagem textual de programação, usando contextos que proveem feedback imediato e uma abordagem de aprendizagem em espiral.

\section{Introdução}

A computação e a comunicação mediada por computador através de redes de computadores afetaram profundamente e estrutura e o funcionamento da sociedade no Século XXI [Castells 2005]. Nesta nova sociedade, conhecimento é chave, especialmente o conhecimento sobre estas tecnologias. A sociedade brasileira, por outro lado, necessita de ações mais eficazes para trazer este conhecimento para o sistema educacional. A Sociedade Brasileira de Computação (SBC) tem contribuído com este objetivo através de ações políticas e acadêmicas para introduzir a computação na educação básica, como é o caso dos Referenciais de Formação em Computação: Educação Básica [SBC 2017].

Nesse mesmo sentido, os acadêmicos brasileiros da área de computação têm produzido, há mais de uma década, centenas de iniciativas de inserção da computação na educação básica [Santos et al. 2018]. Por outro lado, apesar de tantas iniciativas, ainda não há um currículo completo com materiais didáticos para o ensino fundamental. Esta lacuna nos levou a construir um currículo e um conjunto de livros didáticos para o ensino fundamental II do Brasil. Já apresentamos previamente os dois primeiros livros desta iniciativa [Santana et al. 2019, Araujo et al. 2019].

Neste artigo, apresentamos uma terceira parte de nosso currículo e materiais didáticos, agora para o oitavo ano do ensino fundamental. O currículo se baseia nos 
resultados de aprendizagem e eixos temáticos do currículo de referência da CSTA [Seehorn et al. 2011]. Já o livro que agrega nossos materiais didáticos é baseado no uso de ambientes colaborativos online, na reflexão sobre redes sociais online, na robótica educacional com kits de baixo custo, no prosseguimento do uso de linguagens de blocos para o fortalecimento de habilidades de programação e na introdução de uma linguagem de programação textual. Estes conceitos capitalizam sobre conhecimentos prévios através de uma abordagem de aprendizagem em espiral.

\section{Fundamentação Teórica}

A computação impacta a vida dos jovens ao redor do mundo de modo tanto positivo como negativo, como em seu comportamento nas redes sociais. Segundo Boyd e Marwick (2011), embora os jovens participem de diversas redes sociais compartilhando conteúdos, eles não estão dispostos a abrir mão da sua privacidade. Querem ser vistos e compreendidos, não por todos, mas por uma audiência restrita. É evidente que, para que isto ocorra sem transtornos, é necessário compreender os fundamentos tecnológicos das redes.

Um fator positivo que a interação com os computadores desencadeia é o pensamento criativo. Para Siegel (2016), os adolescentes possuem uma predisposição para a criatividade, especialmente através da exploração criativa. A computação se apresenta como um meio para a exploração da criatividade através de programação, lógica, robótica e produção de conteúdo, o que leva à necessidade de formação dos jovens em tópicos de computação. Assim, faz-se necessária a inserção da Computação no ambiente escolar. A robótica tem sido amplamente utilizada devido à sua proximidade dos estudantes e pelo encantamento que os robôs causam. No entanto, esta abordagem não é nova, tendo sido proposta por Papert na década de 1960. Ele afirmava que a Robótica pode contribuir na aprendizagem através do fazer, onde os estudantes são encorajados a descobrir e construir conhecimentos através de atividades práticas [Papert 1986].

Um mapeamento sistemático evidencia um aumento no interesse em utilizar a robótica educacional [Souza et al. 2018]. Geralmente, tais iniciativas visam ao ensino de programação e são comuns nos EUA, mas outros países como o Brasil também possuem suas iniciativas de ensino da Robótica. Esta modalidade tem sido utilizada também para outros fins como a promoção da interdisciplinaridade, a participação em torneios de robótica e o exercício do pensamento computacional, podendo ser aplicada em diversos níveis, do ensino fundamental à graduação. No Brasil, podemos destacar o trabalho de Santos et al. (2016), que apresenta a implantação de um laboratório de robótica para capacitação de estudantes, realização de torneios e produções individuais. Os autores defendem que atividades vinculadas à robótica possibilitam a aplicação de conhecimentos teóricos de forma concreta pelos estudantes. Além disto, estas abordagens podem contribuir para a construção de novas ideias, estimulando a criatividade dos estudantes.

Forte e Guzdial (2004) propõem uma abordagem para minimizar problemas de evasão e reprovação no ensino superior através do ensino de programação pela manipulação de mídias como imagens, áudio e vídeos . Eles apresentam uma abordagem em que estudantes utilizam a linguagem Python com o ambiente Jython Environment for Students (JES) para aprender programação manipulando mídias. Por exemplo, os estudantes manipulam pixels, sobrepondo-os e modificando valores de cores, para criar efeitos como negativos ou escalas de cinza. 
A ferramenta JES também permite a criação de figuras geométricas com a biblioteca Turtle do Python. A Biblioteca Turtle é baseada na Screen Turtle, um simulacro da Floor Turtle criada na década de 1960. A Floor Turtle é um robôtartaruga que tem seu movimento controlado por um computador. Os estudantes controlam a tartaruga com movimentos para frente e para trás, além de rotações em graus para a esquerda e direita. A tartaruga pode riscar o local onde passa, gerando figuras [McNerney 2004]. Através da Turtle, é possível aprender diversos conceitos de programação, como sequência, condicionais, loops e funções. O uso de JES e Python é adequado aos estudantes novatos em programação, o que já foi demonstrado tanto no ensino superior [Forte and Guzdial 2004] como no ensino médio [Araujo et al. 2018] e fundamental [Motta 2010]. Assim como a robótica, figuras geométricas e mídias permitem novas abordagens de ensino-aprendizagem de programação. Todas estas abordagens oferecem aos estudantes conhecimentos relacionados à computação, além de permitir a exploração da criatividade.

\section{Computação e Sociedade: Uma Proposta para o $8^{\circ}$ ano}

Neste trabalho, elaboramos o currículo para o $8^{\circ}$ ano do ensino fundamental, bem como o planejamento das aulas e materiais necessários para a execução deste currículo. Esta é a terceira etapa de um objetivo maior, que é propor um currículo de Computação para o Ensino Fundamental II, do $6^{\circ}$ ao $9^{\circ}$ anos. Na primeira e segunda etapas, que culminaram no programa para o $6^{\circ}$ e $7^{\circ}$ anos, abordamos experiências de aprendizagem projetadas para que os estudantes percebam a computação como uma parte importante de seu mundo, contemplando seus interesses pessoais, e que possam utilizá-la para realizar atividades em pequenas comunidades. Em nossa proposta para o $8^{\circ}$ ano, consideramos que os estudantes estão em uma transição de um foco em pequenos grupos para comportamentos orientados à sociedade. Suas esferas sociais estão crescendo além da família e escola, incluindo a sociedade mais ampla e suas manifestações através de redes sociais online.

No currículo desta série, assim como em nossas propostas para o $6^{\circ}$ e $7^{\circ}$ anos, os resultados de aprendizagem gerais estão definidos em torno de cinco eixos centrais, selecionados a partir do CSTA K-12 Computer Science Standards [Seehorn et al. 2011]. Os eixos trabalhados são: Pensamento Computacional; Práticas de Computação e Programação; Colaboração; Computadores e Dispositivos de Comunicação e Impactos Comunitários, Globais e Éticos. O Eixo Pensamento Computacional está diretamente relacionado à capacidade de resolver problemas. O Eixo Práticas de Computação e Programação envolve a programação e o uso de ferramentas computacionais. O eixo de Colaboração trabalha com a colaboração em diferentes dimensões (estudantes colaboram com colegas e professores, estudantes colaboram a partir de ferramentas online). O Eixo Computadores e Dispositivos de Comunicação explora os elementos dos computadores e dispositivos de comunicação modernos. Por fim, o eixo Impactos Comunitários, Globais e Éticos explora questões éticas relacionadas ao uso do computador tais como uso responsável, respeito aos outros, compartilhamento de informações e questões de equidade. A Figura 1 lista os tópicos escolhidos em cada eixo para a proposta do $8^{\circ}$ ano.

\subsection{Proposta de ensino-aprendizagem para $08^{\circ}$ ano}

Nossa abordagem para o $8^{\circ}$ ano segue o mesmo padrão empregado em nossos trabalhos anteriores para o $6^{\circ}$ e $7^{\circ}$ anos. Consideramos um curso de 60 horas-aula, com carga horária semanal de duas horas. 
IX Congresso Brasileiro de Informática na Educação (CBIE 2020)

Anais do XXVI Workshop de Informática na Escola (WIE 2020)

\section{Computação \& Sociedade}

\section{Pensamento Computacional}

PC2. Usar passos básicos na solução de problemas algorítmicos para projetar soluções .

PC4. Definir um algoritmo como uma sequência de instruções que pode ser processada por um computador.

PC5. Avaliar maneiras pelas quais diferentes algoritmos podem ser usados para resolver o mesmo problema.

PC7. Descrever e analisar uma sequência de instruções que estão sendo seguidas.

PC8. Representar dados de várias formas, incluindo texto, sons, imagens e números.

$\mathrm{PC12}$. Analisar o grau em que um modelo de computador representa com precisão o mundo real.

PC13. Usar abstração para decompor um problema em subproblemas.

PC15. Examinar as conexões entre os elementos da matemática e da ciência da computação, incluindo números binários, lógica, conjuntos e funções.

PC16. Fornecer exemplos de aplicações interdisciplinares do pensamento computacional.

\section{Práticas de Computação e Programação}

PCP3. Usar recursos de tecnologia para a resolução de problemas e o aprendizado auto direcionado.

PCP4. Usar ferramentas de produtividade de finalidade geral e periféricos para apoiar a produtividade pessoal, corrigir déficits de habilidades e facilitar o aprendizado.

PCP6. Usar dispositivos de computação para acessar informações remotas, comunicar-se com outras pessoas e buscar interesses pessoais.

PCP7. Selecionar ferramentas apropriadas e recursos de tecnologia para realizar uma variedade de tarefas e resolver problemas.

PCP8. Usar uma variedade de ferramentas multimídia e periféricos para apoiar a produtividade pessoal e o aprendizado em todo o currículo

PCP9. Projetar, desenvolver, publicar e apresentar produtos usando recursos de tecnologia que demonstram e comunicam conceitos do currículo.

PCP10. Demonstrar compreensão dos algoritmos e sua aplicação prática.

PCP11. Implementar soluções de problemas usando uma linguagem de programação, incluindo: comportamento de looping, instruções condicionais, lógica, expressões, variáveis e funções.

PCP12. Demonstrar boas práticas em segurança de informações pessoais, usando senhas, criptografia e transações seguras.

PCP13. Identificar carreiras interdisciplinares que são aprimoradas pela ciência da computação.

PCP14. Demonstrar disposições para a resolução de problemas abertos e programação.

\section{Colaboração}

C1. Coletar informações e comunicar-se eletronicamente com outras pessoas com suporte de professores, membros da família ou parceiros estudantis.

C2. Usar recursos on-line, como ambientes da Web colaborativos.

C3. Usar ferramentas de tecnologia de produtividade para atividades de redação, comunicação e publicação individuais e colaborativas.

C4. Usar recursos on-line para participar de atividades colaborativas de solução de problemas com o objetivo de desenvolver soluções ou produtos.

C5. Aplicar ferramentas de produtividade/multimídia e periféricos para agrupar a colaboração e apoiar o aprendizado em todo o currículo.

C6. Criar, desenvolver, publicar e apresentar, de forma colaborativa, produtos usando recursos de tecnologia que demonstram e comunicam conceitos do currículo.

C7. Colaborar com colegas, especialistas e outras pessoas usando práticas colaborativas, como programação em pares, trabalho em equipes de projeto e participação em atividades de aprendizado ativo em grupo.

C8. Apresentar as disposições necessárias para colaboração: fornecer feedback útil, integrar feedback, compreender e aceitar múltiplas perspectivas.

\section{Computadores e Disposotovos de Comunicação}

CDC1. Reconhecer que os computadores modelam o comportamento inteligente.

CDC2. Identificar as informações que estão chegando ao computador de várias fontes através de uma rede.

CDC3. Reconhecer que os computadores são dispositivos que executam programas.

CDC4. Identificar uma variedade de dispositivos eletrônicos que contêm processadores computacionais.

CDC5. Demonstrar uma compreensão da relação entre hardware e software.

CDC6. Usar terminologia precisa e adequada ao seu estágio de desenvolvimento ao se comunicar sobre tecnologia.

CDC8. Descrever os principais componentes e funções dos sistemas e redes de computadores.

CDC9. Descrever o que distingue os seres humanos das máquinas, concentrando-se na inteligência humana versus a inteligência das máquinas e nas maneiras pelas quais podemos comunicar.

CDC10. Descrever maneiras pelas quais os computadores usam modelos de comportamento inteligente.

\section{Impactos Comunitários, Globais e Éticos}

IC1. Praticar a cidadania digital responsável (comportamentos legais e éticos) no uso de sistemas de tecnologia e software.

IC2. Identificar comportamentos sociais e éticos positivos e negativos para o uso da tecnologia.

IC3. Discutir questões básicas relacionadas ao uso responsável de tecnologia e informação e as consequências do uso inadequado.

IC4. Identificar o impacto da tecnologia (por exemplo, redes sociais, cyberbullying).

IC5. Compreender questões éticas relacionadas a computadores (por exemplo, direitos autorais e propriedade intelectual).

IC6. Identificar o impacto da tecnologia na vida pessoal e na sociedade.

IC7. Avaliar a precisão, relevância, adequação, abrangência e vieses que ocorrem em fontes de informação eletrônica sobre problemas do mundo real.

IC8. Compreender e descrever questões éticas relacionadas a computadores e redes.

IC9. Expor comportamentos legais e éticos ao usar informações e tecnologia e discutir as consequências do uso indevido.

IC10. Demonstrar conhecimento das mudanças nas tecnologias da informação ao longo do tempo e os efeitos que essas mudanças têm sobre a educação, local de trabalho e sociedade.

IC11. Analisar os impactos positivos e negativos da computação na cultura humana.

Figura 1. Resultados de Aprendizagem para $08^{\circ}$ ano. 


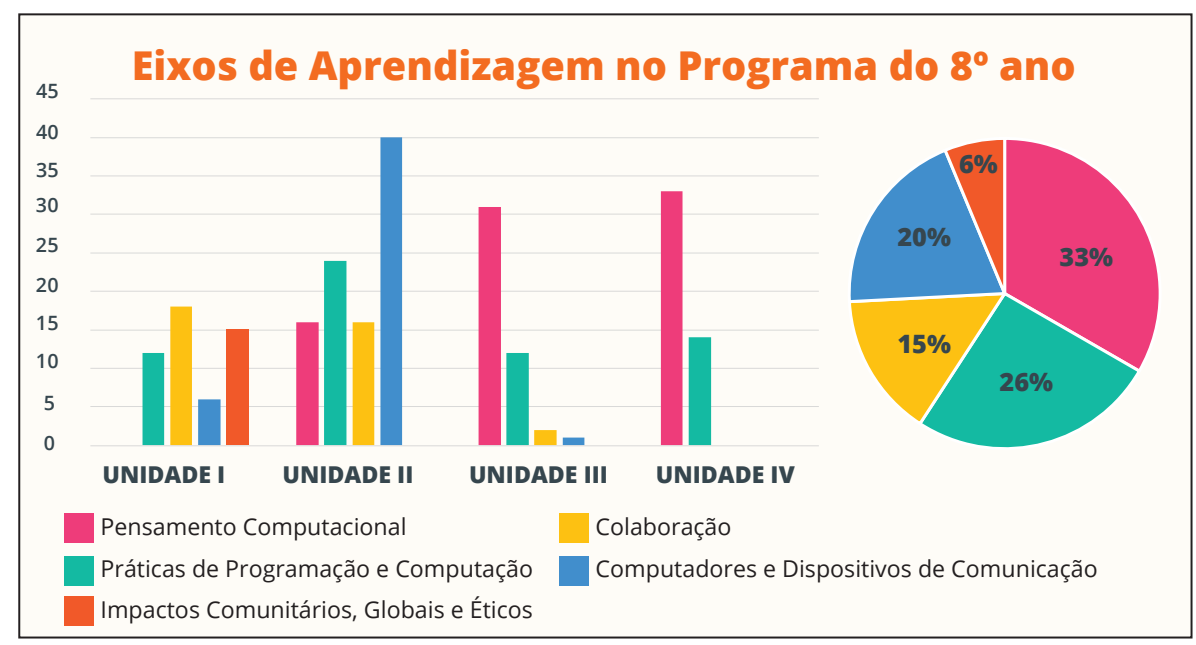

Figura 2. Distribuição dos Resultados de Aprendizagem ao longo do ano.

Elaboramos 30 aulas, divididas em quatro unidades de aprendizagem. Cada unidade possui um tema principal. A Figura 3 lista as aulas de cada unidade, bem como os resultados de aprendizagem esperados e os conteúdos trabalhados. De maneira geral, as aulas propõem diversos tipos de atividades, como programação de robôs, projetos com mídias, atividades de leitura, exercícios escritos, discussão em grupo, dentre outros.

A Unidade I, composta por 8 aulas, tem como tema principal a colaboração por meio de ferramentas online, além de trabalhar com redes de computadores e redes sociais. Os estudantes devem trabalhar com as ferramentas online Google Mail, Drive, Documentos e Apresentações. Outras aulas desta unidade permitem refletir sobre questões éticas relacionadas ao uso das redes sociais e ao compartilhamento de informações online.

A Unidade II, composta por 8 aulas, trabalha com o robô mBot e a ferramenta mBlock da empresa Makeblock ${ }^{1}$. Os estudantes são introduzidos ao universo da robótica e a características dos robôs, como comportamento autônomo. Em cada aula, os estudantes exploram uma funcionalidade diferente do robô mBot, como controle de motores DC, buzzer, LEDs, sensores ultrassônico e de luminosidade e uso do controle remoto IR.

A Unidade III, composta por 7 aulas, busca aprofundar as habilidades de programação dos estudantes e trata de aspectos essenciais da manipulação de imagens. Através da ferramenta GPBlocks, os estudantes exploram o desenvolvimento de filtros de imagens, como preto e branco e o chroma key.

A Unidade IV, composta por 7 aulas, introduz os estudantes à linguagem de programação Python e tem como tema principal desenhos de figuras geométricas através da biblioteca Turtle. Em cada aula, os estudantes exploram os conceitos de programação previamente aprendidos com Scratch, agora usando uma linguagem textual.

A Figura 2 apresenta a distribuição dos tópicos de cada eixo de aprendizagem entre as unidades e a porcentagem com que cada eixo é trabalhado. A categoria Impactos Comunitários, Globais e Éticos aparece apenas na Unidade I, onde reservamos três aulas para trabalhar com os tópicos deste eixo. Pensamento Computacional é o eixo mais

\footnotetext{
${ }^{1}$ Disponível em https://www . makeb lock. com
} 
trabalhado, embora não apareça na Unidade I. O Eixo de Colaboração é trabalhado nas Unidades I e II, com abordagens diferentes. Na Unidade I, trabalhamos com a ideia de colaboração através do uso de ferramentas online. Embora os estudantes trabalhem com seus colegas de classe, queremos tratar da oportunidade que a Internet oferece de trabalhar com pessoas em qualquer lugar do mundo. Na Unidade II, os tópicos de colaboração são abordados com o intuito de trabalhar em grupo para alcançar soluções e ser capaz de dar e receber feedback útil. O eixo Práticas de Programação e Computação aparece de maneira equilibrada ao longo de todo o ano. O eixo Computadores e Dispositivos de Comunicação é trabalhado principalmente na Unidade II, onde os estudantes experimentam com o universo da robótica e criam uma nova percepção sobre sistemas computacionais.

A infraestrutura necessária para implementação de nossa abordagem demanda um laboratório equipado com computadores e robôs mBot, além do acesso à Internet. O professor deve ter conhecimentos básicos na área, incluindo noções de algoritmos, programação em linguagens de bloco e textuais e conceitos básicos de computação.

\subsection{Estrutura do Livro}

O livro Computação e Sociedade ${ }^{2}$ possui duas versões, uma voltada para professores e outra que pode ser distribuída entre os estudantes. É importante salientar que seguimos a mesma organização empregada nas propostas para o $6^{\circ}$ e o $7^{\circ}$ anos. O livro do professor possui diretrizes, instruções gerais e materiais a serem utilizados em sala, enquanto o livro do estudante compila as atividades escritas a serem realizadas em aula e como tarefa para casa. Materiais como mídias e programas-base para os trabalhos no computador são disponibilizados no site de apoio.

O livro do professor oferece um guia completo para os educadores responsáveis por ministrar a disciplina. Cada aula é descrita através de um plano de aula, que inclui os objetivos de aprendizagem, conteúdos trabalhados e as atividades a serem realizadas. Disponibilizamos também um roteiro da aula, que o professor pode seguir se estiver de acordo com nossa abordagem e tiver disponibilidade de todos os recursos necessários. As aulas estão organizadas de acordo com a sequência apresentada na Figura 3.

Cada aula pode conter diferentes seções, a depender do tema e da natureza das atividades. A seção Tópicos Relevantes descreve brevemente alguns dos conceitos empregados na aula e deve apoiar os estudantes durante a execução de suas tarefas. A seção Folha de Atividades possui atividades de escrita ou um guia para as atividades a serem realizadas em sala. A seção Tarefa para Casa é reservada para as atividades extra-classe.

O livro do estudante pode ter em cada aula as seções Tópicos Relevantes, Folha de Tarefas e Tarefa para Casa. Além disso, há um espaço reservado ao final de cada aula para a escrita de um diário de bordo, onde os estudantes devem expressar suas percepções sobre a aula. A principal função do diário de bordo em nossa abordagem é oferecer ao professor feedback útil sobre as experiências de aprendizagem vivenciadas pelos estudantes.

\section{Experiência Preliminar}

Após o desenvolvimento do livro, buscamos aplicar as atividades de modo a avaliar a sua adequação em um ambiente de educação formal. Realizamos uma experiência em uma escola de ensino fundamental no município de Amélia Rodrigues, na Bahia.

\footnotetext{
${ }^{2}$ Disponível em https://sites.google.com/view/computacaofundamental/
} 
IX Congresso Brasileiro de Informática na Educação (CBIE 2020)

Anais do XXVI Workshop de Informática na Escola (WIE 2020)

\begin{tabular}{|c|c|c|}
\hline & AULA & CONTEÚDO \\
\hline \multirow{8}{*}{$\begin{array}{l}\frac{-}{2} \\
\frac{2}{2} \\
\frac{2}{2} \\
\frac{2}{2}\end{array}$} & $\begin{array}{l}1 \text { Redes de Computadores } \\
\mathrm{CDC} 2, \mathrm{CDC5} \text { e CDC8 }\end{array}$ & $\begin{array}{l}\text { Redes de Computadores; Internet; Protocolos de comunicação; } \\
\text { Pacotes de dados; Nós e caminhos; Roteadores; Servidores. }\end{array}$ \\
\hline & $\begin{array}{l}2 \text { Criando um e-mail } \\
\text { PCP4, PCP6, PCP7, PCP12, C1, C4 e IC3 }\end{array}$ & $\begin{array}{l}\text { E-mails; Elementos de um e-mail; Caixa de entrada e caixa de } \\
\text { saída; Serviços de e-mail gratuitos. }\end{array}$ \\
\hline & $\begin{array}{l}3 \text { Editando um Texto no Google Documentos } \\
C D C 1, C D C 2, P C C 5, C 1, C 2 \text { e } C 3\end{array}$ & $\begin{array}{l}\text { Edição no Google Documentos; Colaboração no Google } \\
\text { Documentos; Busca na web. }\end{array}$ \\
\hline & $\begin{array}{l}4 \text { Criando Slides no Google Apresentações } \\
P C P 9, C 1, C 2, C 3 \text { e } C 6\end{array}$ & $\begin{array}{l}\text { Funcionalidades básicas de edição; Funcionalidades de } \\
\text { colaboração; Busca na web. }\end{array}$ \\
\hline & $\begin{array}{l}5 \text { Gerenciando Arquivos no Google Drive } \\
C D C 1, C 1, C 2 \text { e } C 3\end{array}$ & $\begin{array}{l}\text { Computação na Nuvem; Gerenciamento de arquivos no } \\
\text { Google Drive; Colaboração no Google Drive. }\end{array}$ \\
\hline & \begin{tabular}{|l}
6 Redes Sociais \\
PCP3, PCP4, PCP6, PCP8, PCP9, PCP13, C1, C2, C3, C5, C7, IC6, IC10 e IC11
\end{tabular} & $\begin{array}{l}\text { Conceitos e tipos de redes sociais; Importância das redes } \\
\text { sociais na sociedade contemporânea; Internet. }\end{array}$ \\
\hline & $\begin{array}{l}7 \text { Questões éticas em redes sociais } \\
\text { IC1, IC2, IC3, IC6 e IC9 }\end{array}$ & Redes Sociais; Bullying; Stalking; e Respeito às opiniões. \\
\hline & $\begin{array}{l}8 \text { Informações em Redes Sociais } \\
I C 1, I C 2, I C 3, I C 4, I C 6 \text {, IC7 e IC9 }\end{array}$ & $\begin{array}{l}\text { Redes Sociais; Fake news; Atribuição de autoria; } \\
\text { Crimes cibernéticos. }\end{array}$ \\
\hline \multirow{8}{*}{ 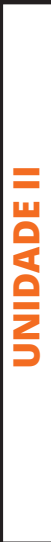 } & $\begin{array}{l}1 \text { Conhecendo o mBot } \\
\mathrm{PC2}, \mathrm{PC7}, \mathrm{PCP} 10, \mathrm{PCP} 11, \mathrm{PCP} 13, \mathrm{C7}, \mathrm{C8}, \mathrm{CDC1}, \mathrm{CDC} 3, \mathrm{CDC4}, \mathrm{CDC9} \text { e CDC10 }\end{array}$ & $\begin{array}{l}\text { Robô mBot; Controle de motores DC; Programação; } \\
\text { o mBlock: eventos, controle, sensores e ação. }\end{array}$ \\
\hline & \begin{tabular}{|l|l|}
2 Fazendo Luz \\
PC2, PC7, PCP10, PCP11, PCP 13, C7, C8, CDC1, CDC3, CDC4, CDC9 e CDC10
\end{tabular} & $\begin{array}{l}\text { Sensores; Sensor de luminosidade; Programação; } \\
\text { o mBlock: eventos, controle, sensores, operadores, mostrar e ação. }\end{array}$ \\
\hline & $\begin{array}{ll}3 & \text { Fazendo a Festa } \\
& \text { PC2, PC7, PCP10, PCP 11, PCP13, C7, C8, CDC1, CDC3, CDC4, CDC9 e CDC10 }\end{array}$ & $\begin{array}{l}\text { Robô mBot; Programação de músicas; Programação; } \\
\text { o mBlock: eventos, controle e mostrar. }\end{array}$ \\
\hline & $\begin{array}{l}4 \text { Evitando obstáculos } \\
\text { PC2, PC7, PCP10, PCP11, PCP13, C7, C8, CDC1, CDC3, CDC4, CDC9 e CDC10 }\end{array}$ & $\begin{array}{l}\text { Robô mBot; Sensor ultrassônico; Programação; mBlock: eventos, } \\
\text { o controle, sensores, operadores, variáveis, mostrar e ação. }\end{array}$ \\
\hline & $\begin{array}{l}5 \text { Carrinho de Controle Remoto } \\
\text { PC2, PC7, } P C P 10, P C P 11, P C P 13, C 7, C 8, C D C 1, C D C 3, C D C 4, C D C 9 \text { e CDC10 }\end{array}$ & $\begin{array}{l}\text { Robô mBot; Controle remoto IR; Programação; mBlock: eventos, } \\
\text { ocontrole, sensores, operadores, variáveis, mostrar e ação. }\end{array}$ \\
\hline & $\begin{array}{l}6 \text { Robô seguidor de linha } \\
\text { PC2, } P C 7, P C P 10, P C P 11, P C P 13, C 7, C 8, C D C 1, C D C 3, C D C 4, C D C 9 \text { e CDC10 }\end{array}$ & $\begin{array}{l}\text { Robô mBot; Sensor seguidor de linha; Programação; mBlock: } \\
\text { oeventos, controle, sensores, operadores e ação. }\end{array}$ \\
\hline & $\begin{array}{l}7 \text { Criando o Robô mais Inteligente do Mundo } \\
\text { PC2, } P C 7, P C P 10, P C P 11, P C P 13, C 7, C 8, C D C 1, C D C 3, C D C 4, C D C 9 \text { e CDC10 }\end{array}$ & $\begin{array}{l}\text { Robô mBot; Sensores; Buzzer; LEDs; motores DC; Programação; } \\
\text { omBlock. }\end{array}$ \\
\hline & 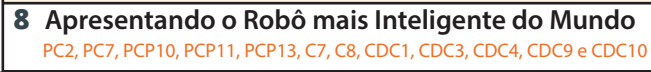 & $\begin{array}{l}\text { Robô mBot; Sensores; Buzzer; LEDs; motores DC; Programação; } \\
\text { o mBlock; Apresentação de Slides. }\end{array}$ \\
\hline \multirow{7}{*}{ 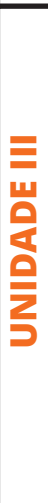 } & $\begin{array}{l}1 \text { Pixel a Pixel } \\
\text { PC2; PC5, PC8, PC12, PC15, PCP10 e PCP11 }\end{array}$ & $\begin{array}{l}\text { Imagens Digitais; Pixels; Padrão de cores RGB; GPBlocks; } \\
\text { Programação: variáaeis, sequência, vetores e matrizes, loops } \\
\text { simples e aninhados, funções de bibliotecas. }\end{array}$ \\
\hline & $\begin{array}{l}2 \text { Efeitos em Imagens Utilizando Matemática } \\
\text { PC2; PC5, PC8, PC12, PC15, PCP10 e PCP11 }\end{array}$ & $\begin{array}{l}\text { Efeitos de imagem; Propriedades dos pixels; GPBlocks; } \\
\text { Programação (mesmos conceitos da aula 1). }\end{array}$ \\
\hline & $\begin{array}{l}3 \text { Mais efeitos utilizando Matemática } \\
\text { PC2; PC5, PC8, PC12, PC15, PCP 10 e PCP } 11\end{array}$ & \\
\hline & $\begin{array}{l}4 \text { Jogo da Laranja (Roteamento e Bloqueio em Redes) } \\
\text { PC16, C7, C8 e CDC8 }\end{array}$ & Roteamento em redes; Bloqueio (deadlock). \\
\hline & \begin{tabular}{|l}
5 Efeitos com Condições \\
PC2; PC5, PC8, PC12, PC15, PCP10 e PCP11
\end{tabular} & $\begin{array}{l}\text { Efeitos de imagem; Propriedades dos pixels; GPBlocks; } \\
\text { Programação (mesmos conceitos da aula 1). }\end{array}$ \\
\hline & $\begin{array}{l}6 \text { Brincando com Chroma key } \\
\text { PC2; PC5, PC8, PC12, PC15, PCP10 e PCP11 }\end{array}$ & \\
\hline & $\begin{array}{l}7 \text { Sendo um Artista com GPBlocks } \\
\text { PC2; PC5, PC8, PC12, PC15, PCP10 e PCP } 11\end{array}$ & \\
\hline \multirow{7}{*}{ 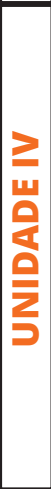 } & $\begin{array}{l}1 \text { Você se Lembra da Caneta? } \\
\text { PC2; PC5, PC7, PC15, PCP10 e PCP } 11\end{array}$ & $\begin{array}{l}\text { Comandos do Scratch: Controle, Movimento e Caneta; } \\
\text { Programação: sequências, loops; Polígonos regulares. }\end{array}$ \\
\hline & $\begin{array}{l}2 \text { Scratch versus Python } \\
\text { PC2; PC5, PC7, PC15, PCP10 e PCP } 11\end{array}$ & $\begin{array}{l}\text { Python; Biblioteca Turtle; Programação em linguagem textual: } \\
\text { sequência e loops. }\end{array}$ \\
\hline & $\begin{array}{l}3 \text { Scratch versus Python (Funções) } \\
\text { PC2; PC5, PC7, PC13, PC15, PCP10 e PCP11 }\end{array}$ & $\begin{array}{l}\text { Python; Biblioteca Turtle; Polígonos regulares; Programação em } \\
\text { linguagem textual: funções. }\end{array}$ \\
\hline & $\begin{array}{l}4 \text { Mais Sobre Loops e Funções em Python } \\
\text { PC2; PC5, PC7, PC13, PC15, PCP10 e PCP11 }\end{array}$ & $\begin{array}{l}\text { Python; Biblioteca Turtle; Polígonos regulares; Programação em } \\
\text { linguagem textual: loops aninhados e funçōes. }\end{array}$ \\
\hline & \begin{tabular}{|l}
5 Mestre da Programação \\
PC2; PC5, PC7, PC13, PC15, PCP10 e PCP11
\end{tabular} & $\begin{array}{l}\text { Python; Biblioteca Turtle; Polígonos regulares; Programação em } \\
\text { linguagem textual: funções simples e funçōes com parâmetros. }\end{array}$ \\
\hline & $\begin{array}{l}6 \text { Condicionais em Python } \\
\text { PC2; PC5, PC7, PC13, PC15, PCP 10 e PCP11 }\end{array}$ & $\begin{array}{l}\text { Python; Biblioteca Turtle; Polígonos regulares; Programação em } \\
\text { linguagem textual: Condicionais. }\end{array}$ \\
\hline & $\begin{array}{l}7 \text { Seja um Artista com Python } \\
\text { PC2; PC5, PC7, PC13, PC15, PCP10 e PCP11 }\end{array}$ & $\begin{array}{l}\text { Python; Biblioteca Turtle; Polígonos regulares; Programação em } \\
\text { linguagem textual: Todos os conceitos explorados. }\end{array}$ \\
\hline
\end{tabular}

Figura 3. Organização das aulas. 
As aulas foram ministradas por um dos autores na disciplina de Informática. Utilizamos o laboratório de informática da escola com 14 computadores, uma lousa branca e conexão à internet. A abordagem contou com a participação de 28 estudantes, sendo 10 meninos e 18 meninas, com idade média de 14, $8 \pm 0,6$ anos. As aulas, semanais, seguiram as atividades propostas no Livro do $8^{\circ}$ ano, utilizando o ambiente JES e a linguagem Python. Aplicamos parcialmente os materiais do livro, mais especificamente, atividades da Unidade IV, em quatro aulas de uma hora de duração cada.

No primeiro dia, os estudantes foram apresentados à linguagem Python e ao JES. Como atividade foi proposta a criação de figuras geométricas como quadrado, triangulo, pentágono e hexágono. No segundo dia, os estudantes realizaram modificações na primeira atividade, adicionando a utilização de funções. Nesta aula, além das figuras geométricas básicas, os estudantes experimentaram combinações de figuras.

No terceiro dia, os estudantes aprenderam a utilizar loops e aplicaram este conceito na criação de figuras mais complexas, a partir da mescla de figuras básicas e modificação da rotação. No quarto dia, os estudantes utilizaram condicionais fazendo variações como a mudança de cor das bordas e preenchimento das figuras.

Apresentamos diversos tópicos sobre programação através de slides e exemplos para elucidar a sintaxe de Python. Utilizamos Turtle inicialmente para criação de figuras geométricas simples, evoluindo para formas mais elaboradas conforme a introdução de novos conceitos. Percebemos que as sequências didáticas planejadas possibilitaram a aplicação do material de forma simples e direta. Sobre as atividades, percebemos que a sequência didática e os exemplos usados facilitaram o entendimento dos estudantes.

\section{Discussão}

Após a nossa experiência preliminar e pelo próprio desenvolvimento do livro didático, algumas lições aprendidas ficaram evidentes, tais como a importância de manter a sensação de familiaridade do estudante com o conteúdo ou ferramenta e a dificuldade com a língua inglesa. A seguir, discutimos brevemente nossas percepções.

A manutenção da familiaridade apoia o processo de aquisição do conhecimento. No livro do $8^{\circ}$ ano, reutilizamos contextos metodológicos previamente utilizados nos anos anteriores, como é o caso da Unidade IV, com Python, que reutiliza a ideia de turtle graphics já trabalhada no ano anterior com Scratch. Do mesmo modo, ao utilizar um novo contexto, como na Unidade III com o contexto de imagens digitais, mantemos a familiaridade usando uma linguagem de blocos. A introdução de novos elementos, sejam linguagens, contextos metodológicos ou ferramentas, deve ser gradual, mantendo a familiaridade através do reuso de conhecimentos prévios. Ausubel já havia utilizado organizadores prévios como suporte para a aprendizagem de novos conceitos, através de suas ideias de aprendizagem significativa, aqui reutilizadas no livro do $8^{\circ}$ ano [Ausubel 1960].

Aprendizagem em espiral permite fortalecer o domínio de conhecimentos prévios. Os materiais desenvolvidos para o $8^{\circ}$ ano reusam conhecimentos já trabalhados nos livros do $6^{\circ}$ e do $7^{\circ}$ anos. Por exemplo, as ideias de sequência, seleção, repetição e modularidade já haviam sido trabalhadas tanto de forma desplugada, no sexto ano, como através de Scratch. Aqui no $8^{\circ}$ ano, elas são retomadas com a utilização de um kit de robótica, na Unidade II, ou servem para apoiar o processamento de imagens, permitindo 
acrescentar conceitos novos como estruturas de dados básicas, ao mesmo tempo em que que os conceitos anteriores são reforçados. Bruner, ao discutir a aprendizagem em espiral, fundamenta esta abordagem em que conhecimentos podem ser apresentados de maneira justa e autêntica em diferentes níveis de complexidade [Bruner 1966], acrescentando, a cada giro da espiral, mais complexidade ao objeto da aprendizagem.

As atividades de complexidade gradual contribuem para a aprendizagem dos estudantes. Usamos uma sequência didática que visa ampliar atividades anteriores, inserindo novos conceitos. Os estudantes são estimulados a criar novas formas a partir de algo já conhecido, revisando conceitos em consolidação. Durante as atividades, não foram encontradas dificuldades quanto à aplicação do conteúdo, sugerindo que a sequência com complexidade gradual contribui para a diminuição da sobrecarga cognitiva.

$O$ inglês é um desafio para o entendimento dos comandos de programação. $\mathrm{Na}$ comunidade em que aplicamos esta experiência, muitos estudantes possuíam pouco ou nenhum conhecimento de inglês. Este problema suscita discussões mais amplas, mas acreditamos que esta é uma realidade comum, principalmente para estudantes de baixa renda. Percebemos que, ao não se sentirem familiarizados com o inglês, muitos tinham dificuldade de associar o texto dos comandos com as ações por eles executadas. Isso dificulta a aprendizagem de programação textual pois os estudantes cometem frequentes erros de digitação, além de não compreenderem erros acusados pelo interpretador.

\section{Conclusões}

Neste artigo, apresentamos uma proposta curricular e um livro didático de computação para o oitavo ano do Ensino Fundamental II, baseados em conceitos e tecnologias de computação e comunicação e no fortalecimento do pensamento computacional. Em uma experiência preliminar, aplicamos uma parte dos materiais do livro em uma escola pública municipal e apresentamos algumas lições aprendidas.

Como síntese das lições aprendidas, destacamos a manutenção da familiaridade com aprendizados prévios, a aprendizagem em espiral como potencializadora da consolidação dos conhecimentos, a complexidade gradual e crescente dos assuntos e as dificuldades que a língua inglesa traz no uso de linguagens de programação textuais.

Com as contribuições deste trabalho, professores podem contar com recursos didáticos concretos para apoiar aulas formais de computação, ao mesmo tempo em que os estudantes utilizam contextos lúdicos e de feedback imediato, permitindo-lhes construir uma reflexão na prática sobre tecnologias contemporâneas e habilidades do século XXI.

Em um futuro próximo, pretendemos avaliar o uso do livro em um ano escolar completo em turmas do oitavo ano do ensino fundamental.

\section{Agradecimentos}

Este projeto foi apoiado pela FAPESP através do auxílio de pesquisa 2015/24331-1 e das bolsas de treinamento técnico 2018/12799-7 e 2018/15614-8.

\section{Referências}

Araujo, L. G. J., Bittencourt, R. A., and Santos, D. M. B. (2018). Contextualized Spiral Learning of Computer Programming in Brazilian Vocational Secondary Education. In FIE 2018 - 48th Annual Frontiers In Education Conference. 
Araujo, L. G. J., Santana, B. L., and Bittencourt, R. A. (2019). Computação e Comunidade: Uma Proposta de Educação em Computação para o Sétimo Ano do Ensino Fundamental II. In WIE 2019 - XXV Workshop de Informática na Escola.

Ausubel, D. P. (1960). The use of advance organizers in the learning and retention of meaningful verbal material. Journal of Educational Psychology, 51(5):267.

Boyd, D. and Marwick, A. E. (2011). Social Privacy in Networked Publics: Teens' Attitudes, Practices, and Strategies. In A decade in Internet Time: Symposium on the Dynamics of the Internet and Society.

Bruner, J. (1966). Uma Nova Teoria de Aprendizagem. Bloch, Rio de Janeiro.

Castells, M. (2005). A Sociedade em Rede, volume 1. Paz e Terra, São Paulo.

Forte, A. and Guzdial, M. (2004). Computers for communication, not calculation: Media as a motivation and context for learning. In 37th Annual Hawaii International Conference on System Sciences.

McNerney, T. S. (2004). From Turtles to Tangible Programming Bricks: Explorations in Physical Language Design. Personal and Ubiquitous Computing, 8(5):326-337.

Motta, M. S. (2010). Contribuições do Superlogo ao Ensino de Geometria. Informática na Educação: Teoria \& Prática, 13(1).

Papert, S. (1986). Constructionism: A New Opportunity for Elementary Science Education. MIT Media Lab.

Santana, B. L., Araujo, L. G. J., and Bittencourt, R. A. (2019). Computação e Eu: Uma Proposta de Educação em Computação para o Sexto Ano do Ensino Fundamental II. In WEI 2019 - XXVII Workshop sobre Educação em Computação.

Santos, O. L., Cury, D., Rafalski, J., and Silveira, P. D. N. (2016). An IOT Computational Robotics Learning Laboratory in Vila Velha, Espírito Santo. In 2016 XI Latin American Conference on Learning Objects and Technology (LACLO), pages 1-6. IEEE.

Santos, P. S., Araujo, L. G. J., and Bittencourt, R. A. (2018). A Mapping Study of Computational Thinking and Programming in Brazilian K-12 Education. In 2018 IEEE Frontiers in Education Conference (FIE).

SBC (2017). Referenciais de Formação em Computação: Educação Básica. http://www.sbc.org.br/files/ ComputacaoEducacaoBasica-versaofinal-julho2017.pdf.

Seehorn, D., Carey, S., Fuschetto, B., Lee, I., Moix, D., O’Grady-Cunniff, D., Owens, B. B., Stephenson, C., and Verno, A. (2011). CSTA K-12 Computer Science Standards: Revised 2011. Technical report, CSTA/ACM, New York, NY, USA. 10411.

Siegel, D. J. (2016). Cérebro Adolescente: O Grande Potencial, a Coragem e a Criatividade da Mente dos 12 aos 24 anos. Versos, São Paulo.

Souza, I. M., Andrade, W. L., Sampaio, L. M., and Araujo, A. L. S. O. (2018). A Syste-

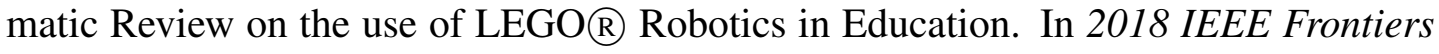
in Education Conference (FIE), pages 1-9. IEEE. 